\title{
Quantitative unique continuation for a parabolic equation
}

\author{
Guher Camliyurt and Igor Kukavica
}

Friday $9^{\text {th }}$ November, 2018

\begin{abstract}
Department of Mathematics, University of Southern California, Los Angeles, CA 90089
e-mails: camliyur@usc.edu, kukavica@usc.edu
\end{abstract}

\begin{abstract}
We address the quantitative uniqueness properties of the solutions of the parabolic equation $\partial_{t} u-\Delta u=w_{j}(x, t) \partial_{j} u+v(x, t) u$ where $v$ and $w$ are bounded. We prove that for solutions $u$, the order of vanishing is bounded by $C\left(\|v\|_{L^{\infty}}^{2 / 3}+\|w\|_{L^{\infty}}^{2}\right)$ matching the upper bound previously established in the elliptic case.
\end{abstract}

\section{Introduction}

The main purpose of this paper is to provide quantitative uniqueness estimates for a parabolic equation

$$
\partial_{t} u-\Delta u=w_{j}(x, t) \partial_{j} u+v(x, t) u
$$

by finding an upper bound for the order of vanishing of a solution in terms of the size of coefficients $v$ and $w$.

Starting with the works of Carleman [C], there have been many results showing that solutions of the equations of parabolic or elliptic type have a finite order of vanishing, in which case we say that the equation has the unique continuation property. For instance, a result of Escauriaza and Vega [EV] shows that for $v$ in a sharp space $L_{t}^{\infty} L_{x}^{n / 2}$ (with the natural smallness assumption for the corresponding norm) and $w=0$, the equation (1.1) has the unique continuation property. Also, Koch and Tataru proved in [KT] that the equation (2.1) has this property provided $v$ and $w$ belong to appropriate Lebesgue spaces. A sharp result of this type for the elliptic counterpart was established by Jerison and Kenig [JK] (cf. $\mathrm{K} 1, \mathrm{~K} 2, \mathrm{~K} 3, \mathrm{~V}$ ) for reviews on unique continuation for elliptic, parabolic, and dispersive equations).

More recently, considerable efforts were dedicated to the quantitative estimates of unique continuation, i.e., to estimating the maximal degree to which the solution may vanish at a point. There are many applications of such results; in particular, quantitative uniqueness results yield lower bounds on solutions of the corresponding PDE. Also, estimates on the order of vanishing are an essential tool for obtaining upper bounds on the size of level sets of PDE [DF1, DF2, DF3, Ku2, Ku3, Ku4, L, in inverse problems and control $\mathrm{AE}$, upper bounds on vortex degrees [Ku2, spectral information on Schrodinger operators [BK, backward uniqueness [EF, Hadamard type theorems [AMRV, B1, B2, BC, CRV, EFV, EVe, LNW], and other topics. 
The research on quantitative uniqueness was initiated by Donnelly and Fefferman [DF1, DF2, DF3, who proved that the maximal order of vanishing $\mathcal{O}_{u}$ of an eigenfunction $u$ of an elliptic operator is bounded by $C \sqrt{\lambda}$, where $\lambda$ is the corresponding eigenvalue. They used this estimate to prove that the nodal volume of a zero set of an analytic eigenfunction is bounded by $C \sqrt{\lambda}$. It is natural to ask the following question: Is the order of vanishing of a solution of a boundary value problem $-\Delta u=w \nabla u+v u$ subject to periodic, Dirichlet, or Neumann conditions (or with prescribed constant doubling property) bounded by

$$
C\|v\|_{L^{\infty}}^{1 / 2}+C\|w\|_{L^{\infty}}
$$

Ku1. This question remains open and a precise upper bound is currently unknown. Based on an example by Meshkov [M], who provided a complex valued solution decaying with a certain exponential rate at infinity, Bourgain and Kenig predicted that (when $w=0$ ), the optimal vanishing rate is more likely $C\|v\|_{L^{\infty}}^{2 / 3}$. In addition, they proved that the order of vanishing can be estimated by this quantity for any solution of the elliptic equation $\partial_{i}\left(a_{i j} \partial_{j} u\right)+v u=0$ (cf. also [KSW] for stronger results in the plane).

In this paper, we address this question for the parabolic equation (1.1). As it is the case for the elliptic equation, it is an open problem whether, under natural growth conditions at infinity (for example as those in the present paper), the quantity (1.2) provides an upper bound for the order of vanishing for solutions $u$ of (1.1). Our main result asserts that the order of vanishing bounded by

$$
C\|w\|_{L^{\infty}}^{2}+C\|v\|_{L^{\infty}}^{2 / 3} .
$$

This result is in agreement with an elliptic upper bound proved by Bourgain and Kenig in [BK when $w=0$. However, our proof is based on a completely completely different approach, which we describe next.

The method presented here is based on the parabolic frequency function introduced by Kurata Kur and Poon $[\mathrm{P}$, which is in turn inspired by earlier works on elliptic equations by Almgren [Al] and Garofalo and Lin GL]. It is based on the observation that the frequency

$$
\phi(t)=\int u^{2}(x, t) G_{0}(x, t) d x
$$

is logarithmically convex for solutions of the heat equation. Above,

$$
G_{0}(x, t)=\frac{1}{t^{n / 2}} e^{|x|^{2} / 4 t}, \quad x \in \mathbb{R}^{n}, \quad t>0
$$

denotes the $(2 \pi)^{n / 2}$-multiple of a backward Gaussian kernel. It was also shown in $\mathrm{Kur}$, P that the method can be applied to the equation (1.1) yielding a strong unique continuation property for $u$ when $v$ and $w$ are bounded. Following the dependence on $\|v\|_{L^{\infty}}$ and $\|w\|_{L^{\infty}}$, we obtain that the degree of vanishing (for example under an assumption of periodicity) is bounded by

$$
C\|v\|_{L^{\infty}}^{2}+C e^{C\|w\|_{L^{\infty}}} .
$$

In order to improve this result and obtain the bound (1.3), we use a similarity variable approach from Ku2 (cf. also $\mathrm{An}, \mathrm{Ch}$ ) with the addition of a change of variable introduced in Ku4 which optimizes the Dirichlet quotient (i.e., replaces $Q$ in (4.6) with $\bar{Q}$ in (4.11)). The similarity change of variable was 
used in many contexts; here the idea is that the parabolic structure and the Dirichlet quotient method ([A, AN, CFNT, FS]) leads to the necessary logarithmic convexity. In addition, we use in an essential way theorems due to Alessandrini and Vessella $[\mathrm{AV}$, on the polynomial approximation of a solution of a parabolic equation (cf. also [H1, H2]).

The paper is structured as follows. In Section 2 we state the main result on the maximal order of vanishing for the equation (1.1). In Section 3 we find a point $x_{\epsilon}$ and the time $-\epsilon$, where in a certain sense the frequency is optimized. In Section 4 we recall the similarity change of variables and spectral properties of the resulting linear part. Finally, Section 5 contains the proof of the main theorem.

\section{Notation and the main result}

Our goal is to address the quantitative uniqueness for solutions $u$ of the equation

$$
\partial_{t} u-\Delta u=w_{j}(x, t) \partial_{j} u+v(x, t) u
$$

defined for $(x, t) \in \mathbb{R}^{n} \times I$, where $I$ is an open interval containing $\left[T_{0}, T_{0}+T\right]$, with $T_{0} \in \mathbb{R}$ and $T>0$. The solution $u$ as well as the coefficients $v, w \in L^{\infty}\left(\mathbb{R}^{n}\right)$ are assumed to be periodic in $x$ with respect to $\Omega=[-\pi, \pi]^{n}$ (for the conditions omitting the periodicity assumption, cf. Remark 2.2 and Section 6 below). Also, assume that

$$
\left|w_{j}(x, t)\right| \leq M_{1}, \quad(x, t) \in \mathbb{R}^{n} \times I, \quad j=1, \ldots, n
$$

and

$$
|v(x, t)| \leq M_{0}, \quad(x, t) \in \mathbb{R}^{n} \times I .
$$

Since we are interested in the dependence of the order of vanishing on $M_{0}$ and $M_{1}$ when they are large, we assume $M_{0}, M_{1} \geq 1$. For any $\left(x_{0}, t_{0}\right) \in \mathbb{R}^{n} \times \mathbb{R}$ and $r>0$, denote by

$$
Q_{r}\left(x_{0}, t_{0}\right)=\left\{(x, t) \in \mathbb{R}^{n} \times \mathbb{R}:\left|x-x_{0}\right|<r,-r^{2}<t-t_{0}<0\right\},
$$

the parabolic cylinder centered at $\left(x_{0}, t_{0}\right)$ with radius $r>0$, while the parabolic norm of $(x, t) \in \mathbb{R}^{n} \times \mathbb{R}$ is given by

$$
|(x, t)|=\left(|x|^{2}+|t|\right)^{1 / 2} .
$$

We write $W_{\infty}^{2,1}\left(Q_{1}\right)$ for the Sobolev space of functions whose $x$-derivatives up to second order and $t$ derivative up to the first order belong to $L^{\infty}\left(Q_{1}\right)$.

Denote by $O_{\left(x_{0}, t_{0}\right)}(u)$ the order of vanishing of $u$ at $\left(x_{0}, t_{0}\right)$, which we define (in the $L^{2}$ sense) as the smallest integer $d$ such that

$$
\|u\|_{L^{2}\left(Q_{r}\left(x_{0}, t_{0}\right)\right)}=\mathcal{O}\left(r^{d+(n+2) / 2}\right), \quad \text { as } r \rightarrow 0^{+} .
$$

Let $q_{0}$ be an upper bound for the Dirichlet quotients of $u$ on $\left[T_{0}, T_{0}+T\right]$, i.e.,

$$
\frac{\|\nabla u(\cdot, t)\|_{L^{2}}^{2}}{\|u(\cdot, t)\|_{L^{2}}^{2}} \leq q_{0}, \quad t \in\left[T_{0}, T_{0}+T\right] .
$$

The following is the main theorem of this paper. 
Theorem 2.1. Let $u \in W_{\infty}^{2,1}(\Omega \times I)$ be a nontrivial solution of (2.1) for $t$ in a neighborhood of $\left[T_{0}, T_{0}+T\right]$ with $w_{j}$ and $v$ satisfying (2.2) and (2.3), respectively. Then the order of vanishing of $u$ at $\left(x_{0}, t_{0}\right)$ satisfies

$$
O_{\left(x_{0}, t_{0}\right)}(u) \leq C\left(M_{1}^{2}+M_{0}^{2 / 3}\right)
$$

for every $\left(x_{0}, t_{0}\right) \in \Omega \times\left[T_{0}+T / 2, T_{0}+T\right]$ where the constant $C$ depends on $T, L$, and $q_{0}$.

Remark 2.2. It is not difficult to check that it is possible to replace the $x$-periodicity assumption with

$$
\int_{\mathbb{R}^{n}} u(x, t)^{2} d x \leq M \int_{B_{1}} u(x, t)^{2} d x, \quad t \in\left[T_{0}, T_{0}+T\right]
$$

where $M$ is a constant, making an assertion about the doubling at the point $\left(0, T_{0}+T\right)$. For the necessary modifications, cf. Section 6 below.

The proof of Theorem 2.1 is divided into several lemmas. Let $\left(x_{0}, t_{0}\right) \in \Omega \times\left[T_{0}+T / 2, T_{0}+T\right]$. By translation and rescaling, we may assume that $u$ is defined for $t$ in an open interval $I$ of $[-T, 0]$ and that

$$
\left(x_{0}, t_{0}\right)=(0,0)
$$

\section{Optimizing the frequency function}

Recall from (1.5) that $G_{0}$ represents the $(4 \pi)^{n / 2}$-multiple of the backward Gaussian kernel.

Lemma 3.1. Let $u$ be as above with $v$ and $w_{j}$ satisfying (2.2) and (2.3). Assume that $\epsilon \in(0, T)$. Then

$$
\frac{\epsilon \int_{\mathbb{R}^{n}}\left|\nabla u\left(x_{\epsilon}+y,-\epsilon\right)\right|^{2} G_{0}(y,-\epsilon) d y}{\int_{\mathbb{R}^{n}} u\left(x_{\epsilon}+y,-\epsilon\right)^{2} G_{0}(y,-\epsilon) d y} \leq \epsilon q(-\epsilon)
$$

for some $x_{\epsilon} \in \Omega$.

This lemma shall be used below with $\epsilon=1 / C\left(M_{1}^{2}+M_{0}^{2 / 3}\right)$ where $C$ is sufficiently large.

Proof of Lemma 3.1. Assume that, for some $\lambda>0$, we have

$$
\lambda \int_{\mathbb{R}^{n}} u(x+y,-\epsilon)^{2} G_{0}(y,-\epsilon) d y \leq \int_{\mathbb{R}^{n}}|\nabla u(x+y,-\epsilon)|^{2} G_{0}(y,-\epsilon) d y, \quad x \in \mathbb{R}^{n} .
$$

We intend to show that this is not possible if $\lambda>q(-\epsilon)$ (this is sufficient since due to periodicity and continuity, the minimum of the quotient in (3.1) is achieved). The statement (3.2) is equivalent to

$$
\int_{\mathbb{R}^{n}} u(y,-\epsilon)^{2} G_{0}(y-x,-\epsilon) d y \leq \frac{1}{\lambda} \int_{\mathbb{R}^{n}}|\nabla u(y,-\epsilon)|^{2} G_{0}(y-x,-\epsilon) d y, \quad x \in \mathbb{R}^{n} .
$$


Integrating the left side of (3.3) over $\Omega$ and using $\int_{\mathbb{R}^{n}} G_{0}(y-x,-\epsilon) d x=(4 \pi)^{n / 2}$, we obtain

$$
\begin{aligned}
& \int_{\Omega} \int_{\mathbb{R}^{n}} u(y,-\epsilon)^{2} G_{0}(y-x,-\epsilon) d y d x=\int_{\Omega} \sum_{j \in \mathbb{Z}^{n}} \int_{j+\Omega} u(y,-\epsilon)^{2} G_{0}(y-x,-\epsilon) d y d x \\
& =\int_{\Omega} \sum_{j \in \mathbb{Z}^{n}} \int_{\Omega} u\left(y^{\prime},-\epsilon\right)^{2} G_{0}\left(y^{\prime}+j-x,-\epsilon\right) d y^{\prime} d x \\
& \quad=\int_{\Omega} u\left(y^{\prime},-\epsilon\right)^{2}\left(\sum_{j \in \mathbb{Z}^{n}} \int_{\Omega} G_{0}\left(y^{\prime}+j-x,-\epsilon\right) d x\right) d y^{\prime} \\
& =\int_{\Omega} u\left(y^{\prime},-\epsilon\right)^{2} \int_{\mathbb{R}^{n}} G_{0}\left(y^{\prime}-x,-\epsilon\right) d x d y^{\prime} \\
& =(4 \pi)^{n / 2} \int_{\Omega} u\left(y^{\prime},-\epsilon\right)^{2} d y^{\prime} .
\end{aligned}
$$

Similarly, we have

$$
\int_{\Omega} \int_{\mathbb{R}^{n}}|\nabla u(y,-\epsilon)|^{2} G_{0}(y-x,-\epsilon) d y d x=(4 \pi)^{n / 2} \int_{\Omega}\left|\nabla u\left(y^{\prime},-\epsilon\right)\right|^{2} d y^{\prime} .
$$

Combining (3.4) and (3.5) with (3.3), we get

$$
\lambda \int_{\Omega} u(y,-\epsilon)^{2} d y \leq \int_{\Omega}|\nabla u(y,-\epsilon)|^{2} d y=q(-\epsilon) \int_{\Omega} u(y,-\epsilon)^{2} d y .
$$

Since $u(\cdot,-\epsilon)$ is not identically zero, we obtain a contradiction if $\lambda>q(-\epsilon)$. Setting $\lambda=2 q(-\epsilon)$, we conclude that there exists $x_{\epsilon} \in \mathbb{R}^{n}$ such that (3.1) holds. By periodicity of $u$, we may assume that $x_{\epsilon} \in \Omega$.

We proceed with a change of variables so that at time $-\epsilon$ the solutions starts at the point $x_{\epsilon}$ from Lemma 3.1 Let

$$
u(x, t)=\widetilde{u}\left(x-\frac{x_{\epsilon}}{\epsilon} t, t\right) .
$$

We then have

$$
\widetilde{u}(x,-\epsilon)=u\left(x+x_{\epsilon},-\epsilon\right)
$$

and

$$
\widetilde{u}(x, 0)=u(x, 0) .
$$

Note also that

$$
O_{(0,0)}(\widetilde{u})=O_{(0,0)}(u)
$$

Furthermore,

$$
\frac{\int_{\Omega}|\nabla \widetilde{u}(x, t)|^{2} d x}{\int_{\Omega} \widetilde{u}(x, t)^{2} d x}=\frac{\int_{\Omega}|\nabla u(x, t)|^{2} d x}{\int_{\Omega} u(x, t)^{2} d x}=q(t),
$$


for all $t \in[-\epsilon, 0)$, and (3.1) may be written as

$$
\frac{\epsilon \int_{\mathbb{R}^{n}}|\nabla \widetilde{u}(y,-\epsilon)|^{2} G_{0}(y,-\epsilon) d y}{\int_{\mathbb{R}^{n}} \widetilde{u}(y,-\epsilon)^{2} G_{0}(y,-\epsilon) d y} \leq \epsilon q(-\epsilon) .
$$

The function $\widetilde{u}$ solves a modified equation

$$
\partial_{t} \widetilde{u}-\Delta \widetilde{u}=-\frac{1}{\epsilon} x_{\epsilon} \cdot \nabla \widetilde{u}+w \cdot \nabla \widetilde{u}+v \widetilde{u} .
$$

Observing (3.10), we remove tilde from here on, and write $u$ instead of $\widetilde{u}$. Setting

$$
a=-\frac{x_{\epsilon}}{\epsilon},
$$

(3.13) may then be rewritten as

$$
\partial_{t} u-\Delta u=a_{j} \partial_{j} u+w_{j} \partial_{j} u+v u .
$$

\section{Similarity variables}

Next, we apply a similarity change of variable

$$
u(x, t)=e^{|x|^{2} / 8(-t)} U\left(\frac{x}{\sqrt{-t}},-\log (-t)\right)
$$

i.e.,

$$
U(y, \tau)=e^{-|y|^{2} / 8} u\left(y e^{-\tau / 2},-e^{-\tau}\right)
$$

with $\tau=-\log (-t)$, i.e., $t=-e^{-\tau}$ for $t \in[-\epsilon, 0]$. Also, write

$$
V(y, \tau)=v\left(y e^{-\tau / 2},-e^{-\tau}\right), \quad(y, \tau) \in \mathbb{R}^{n} \times\left[\tau_{0}, \infty\right)
$$

and

$$
W_{j}(y, \tau)=w_{j}\left(y e^{-\tau / 2},-e^{-\tau}\right), \quad(y, \tau) \in \mathbb{R}^{n} \times\left[\tau_{0}, \infty\right),
$$

for $j=1, \ldots, n$ where $\tau_{0}=\log (1 / \epsilon)$. Denoting

$$
H U=-\Delta U+\left(\frac{|y|^{2}}{16}-\frac{n}{4}\right) U
$$

and

$$
Q(\tau)=\frac{(H U, U)_{L^{2}\left(\mathbb{R}^{n}\right)}}{\|U\|_{L^{2}\left(\mathbb{R}^{n}\right)}^{2}}
$$

the equation (3.14) may be written as

$$
\partial_{\tau} U+H U=e^{-\tau / 2}\left(a_{j} y_{j} U+a_{j} \partial_{j} U\right)+e^{-\tau / 2}\left(y_{j} W_{j} U+W_{j} \partial_{\tau} U\right)+e^{-\tau} V U
$$


for $\tau \geq \tau_{0}$, while at $\tau=\tau_{0}$,

$$
U\left(y, \tau_{0}\right)=e^{-|y|^{2} / 8} u\left(\frac{y}{\sqrt{\epsilon}},-\epsilon\right)
$$

Then

$$
\begin{aligned}
\partial_{\tau} U & +(A(\tau)-\bar{Q}(\tau) I) U+\bar{Q}(\tau) U \\
& =e^{-\tau / 2} a_{j} \partial_{j} U+e^{-\tau / 2}\left(y_{j} W_{j} U+W_{j} \partial_{j} U\right)+e^{-\tau} V U
\end{aligned}
$$

where we denote

$$
A(\tau) U=H U-e^{-\tau / 2} a_{j} y_{j} U
$$

and

$$
\bar{Q}(\tau)=\frac{(A(\tau) U, U)_{L^{2}\left(\mathbb{R}^{n}\right)}}{\|U\|_{L^{2}\left(\mathbb{R}^{n}\right)}^{2}}=Q(\tau)-\frac{e^{-\tau / 2} a_{j}}{\|U\|_{L^{2}\left(\mathbb{R}^{n}\right)}^{2}} \int_{\mathbb{R}^{n}} y_{j} U^{2} d y .
$$

A straight-forward change of variables yields

$$
\|U(\cdot, \tau)\|_{L^{2}\left(\mathbb{R}^{n}\right)}^{2}=\int_{\mathbb{R}^{n}} u(x, t)^{2} G_{0}(x, t) d x
$$

and

$$
(H U(\cdot, \tau), U(\cdot, \tau))_{L^{2}\left(\mathbb{R}^{n}\right)}=|t| \int_{\mathbb{R}^{n}}|\nabla u(x, t)|^{2} G_{0}(x, t) d x .
$$

We now observe a simple fact

$$
\|(H-\bar{Q}(\tau) I) v\|_{L^{2}\left(\mathbb{R}^{n}\right)} \geq \operatorname{dist}(\bar{Q}(\tau), \operatorname{sp}(H)), \quad\|v\|_{L^{2}\left(\mathbb{R}^{n}\right)}=1 .
$$

In order to analyze the asymptotic behavior of eigenvalues of $A(\tau)$, we first recall the spectral properties of $H$. The Hermite polynomials $h_{k}$ are defined on the real line as

$$
h_{k}(x)=(-1)^{k} e^{x^{2}} \frac{d^{k}}{d x^{k}} e^{-x^{2}},
$$

while the Hermite functions read

$$
\widetilde{h}_{k}(x)=h_{k}(x) e^{-x^{2} / 2} .
$$

By taking the product of one-dimensional Hermite functions, we generalize the definition to $\mathbb{R}^{n}$,

$$
\phi_{\alpha}(x)=\prod_{k=1}^{n} \widetilde{h}_{\alpha_{k}}\left(x_{k}\right),
$$

where $\alpha=\left(\alpha_{1}, \ldots, \alpha_{n}\right) \in \mathbb{N}_{0}^{n}$, and $x=\left(x_{1}, \ldots, x_{n}\right) \in \mathbb{R}^{n}$. The set

$$
\left\{\phi_{\alpha}: \alpha \in \mathbb{N}_{0}^{n}\right\}
$$


forms a complete orthonormal system for $L^{2}\left(\mathbb{R}^{n}\right)$ and the functions $\phi_{\alpha}$ solve

$$
\left(\Delta-|x|^{2}\right) \phi_{\alpha}(x)=-(2|\alpha|+n) \phi_{\alpha} .
$$

Then we have

$$
H\left(\phi_{\alpha}\left(\frac{x}{2}\right)\right)=\frac{|\alpha|}{2} \phi_{\alpha}\left(\frac{x}{2}\right), \quad \alpha \in \mathbb{N}_{0}^{n}
$$

and thus

$$
\operatorname{sp}(H)=\left\{\frac{m}{2}: m \in \mathbb{N}_{0}\right\} .
$$

Lemma 4.1. For a sufficiently large constant $K_{0}>0$, set

$$
\epsilon=\frac{1}{K_{0}\left(M_{1}^{2}+M_{0}^{2 / 3}\right)} .
$$

Then

$$
\bar{Q}(\tau)=\mathcal{O}\left(\frac{1}{\epsilon}\right), \text { as } \tau \rightarrow \infty
$$

Also,

$$
\bar{Q}(\tau) \rightarrow \frac{m}{2}, \quad \text { as } \tau \rightarrow \infty
$$

for some $m \in \mathbb{N}_{0}$ for which

$$
m \leq C\left(M_{0}^{2 / 3}+M_{1}^{2}\right)
$$

where $C$ depends on $q_{0}$. Moreover, for every $\delta>0$, there exist $\eta \in(\log (1 / \epsilon), 0)$ and constants $A_{1}(\delta), A_{2}(\delta)>$ 0 such that

$$
A_{1}(\delta)|t|^{m+\delta} \leq \int u^{2}(x, t) G_{0}(x, t) d x \leq A_{2}(\delta)|t|^{m-\delta}
$$

for all $t \in[-\eta, 0)$.

Proof of Lemma 4.1. We first divide (4.9) by $\|U\|_{L^{2}\left(\mathbb{R}^{n}\right)}$ and then take the inner product of the resulting equation with $(A(\tau)-\bar{Q}(\tau) I) U /\|U\|_{L^{2}\left(\mathbb{R}^{n}\right)}$. Let $B(U)$ denote the right side of (4.9). We obtain

$$
\begin{aligned}
& \left(\frac{\partial_{\tau} U}{\|U\|},(A(\tau)-\bar{Q}(\tau) I) \frac{U}{\|U\|}\right)+\left\|(A(\tau)-\bar{Q}(\tau) I) \frac{U}{\|U\|}\right\|^{2}+\bar{Q}(\tau) \frac{(U, A(\tau) U)}{\|U\|^{2}} \\
& \quad=\bar{Q}^{2}(\tau)+\left(\frac{B(U)}{\|U\|},(A(\tau)-\bar{Q}(\tau) I) \frac{U}{\|U\|}\right)
\end{aligned}
$$

where we abbreviate

$$
(\cdot, \cdot)=(\cdot, \cdot)_{L^{2}\left(\mathbb{R}^{n}\right)}
$$

and

$$
\|\cdot\|=\|\cdot\|_{L^{2}\left(\mathbb{R}^{n}\right)} \cdot
$$


Note that

$$
\bar{Q}(\tau) \frac{(U, A(\tau) U)}{\|U\|^{2}}=\bar{Q}^{2}(\tau)
$$

so (4.25) becomes

$$
\begin{gathered}
\left(\frac{\partial_{\tau} U}{\|U\|},(A(\tau)-\bar{Q}(\tau) I) \frac{U}{\|U\|}\right)+\left\|(A(\tau)-\bar{Q}(\tau) I) \frac{U}{\|U\|}\right\|^{2} \\
=\left(\frac{B(U)}{\|U\|},(A(\tau)-\bar{Q}(\tau) I) \frac{U}{\|U\|}\right) .
\end{gathered}
$$

On another note, by differentiating (4.11), we get

$$
\bar{Q}^{\prime}(\tau)=\left(2 \frac{\left(\partial_{\tau} U, A(\tau) U\right)}{\|U\|^{2}}+\frac{\left(U, A^{\prime}(\tau) U\right)}{\|U\|^{2}}-\frac{2\left(\partial_{\tau} U, U\right)(A(\tau) U, U)}{\|U\|^{4}}\right) .
$$

Since $A(\tau)$ is a symmetric operator whose derivative is given by

$$
A^{\prime}(\tau) U=\frac{1}{2} e^{-\tau / 2} a_{j} y_{j} U
$$

we obtain

$$
\begin{aligned}
\frac{1}{2} \bar{Q}^{\prime}(\tau) & =\frac{\left(\partial_{\tau} U, A(\tau) U\right)}{\|U\|^{2}}+\frac{1}{4} e^{-\tau / 2} \frac{\left(U, a_{j} y_{j} U\right)}{\|U\|^{2}}-\frac{\left(\partial_{\tau} U, U\right)(A(\tau) U, U)}{\|U\|^{4}} \\
& =\frac{\left(\partial_{\tau} U,(A(\tau)-\bar{Q}(\tau) I) U\right)}{\|U\|^{2}}+\frac{1}{4} e^{-\tau / 2} \frac{\left(U, a_{j} y_{j} U\right)}{\|U\|^{2}} .
\end{aligned}
$$

Then, substituting (4.30) in (4.27), we get

$$
\begin{aligned}
\frac{1}{2} \bar{Q}^{\prime}(\tau)+ & \left\|(A(\tau)-\bar{Q}(\tau) I) \frac{U}{\|U\|}\right\|^{2} \\
= & \frac{1}{4} e^{-\tau / 2} \frac{\left(a_{j} y_{j} U, U\right)}{\|U\|^{2}}+\frac{e^{-\tau / 2} a_{j}}{\|U\|^{2}}\left(\partial_{j} U,(A(\tau)-\bar{Q}(\tau) I) U\right) \\
& +\frac{1}{\|U\|^{2}}\left(e^{-\tau / 2}\left(y_{j} W_{j} U+W_{j} \partial_{j} U\right)+e^{-\tau} V U,(A(\tau)-\bar{Q}(\tau) I) U\right) .
\end{aligned}
$$

We integrate by parts on the second term on the right hand side and note that $\int U \partial_{j} U=0$ and $\int \Delta U \partial_{j} U=0$. Therefore, (4.31) becomes

$$
\begin{aligned}
\frac{1}{2} \bar{Q}^{\prime}(\tau)+ & \left\|(A(\tau)-\bar{Q}(\tau) I) \frac{U}{\|U\|}\right\|^{2} \\
= & \frac{e^{-\tau / 2} a_{j}}{4\|U\|^{2}} \int y_{j} U^{2} d y-\frac{e^{-\tau / 2} a_{j}}{16\|U\|^{2}} \int y_{j} U^{2} d y+\frac{e^{-\tau}|a|^{2}}{2} \\
& \quad+\frac{e^{-\tau / 2}}{\|U\|^{2}} \int\left(y_{j} W_{j} U+W_{j} \partial_{j} U+e^{-\tau / 2} V U\right)(A(\tau)-\bar{Q}(\tau) I) U d y . \\
= & I_{1}+I_{2}+I_{3}+I_{4} .
\end{aligned}
$$

Now,

$$
\begin{aligned}
I_{1} & =\frac{e^{-\tau / 2}}{4\|U\|^{2}} a_{j} \int y_{j} U^{2} d y \leq \frac{e^{-\tau / 2}}{4\|U\|^{2}}|a| \int|y| U^{2} d y \\
& \leq \frac{e^{-\tau / 2}}{4\|U\|^{2}}|a|\left(\int|y|^{2} U^{2} d y\right)^{1 / 2},
\end{aligned}
$$


and similarly

$$
I_{2}+I_{3} \leq \frac{e^{-\tau / 2}}{16\|U\|}|a|\left(\int|y|^{2} U^{2} d y\right)^{1 / 2}+\frac{e^{-\tau}|a|^{2}}{2} .
$$

As for the last term in (4.32),

$$
I_{4} \leq\left\|(A(\tau)-\bar{Q}(\tau) I) \frac{U}{\|U\|}\right\|\left(\frac{e^{-\tau / 2}}{\|U\|}\left\|y_{j} W_{j} U+W_{j} \partial_{j} U\right\|+e^{-\tau}\|V U\|\right) .
$$

Hence, for the right side of (4.32) we have

$$
\begin{aligned}
I_{1}+I_{2}+ & I_{3}+I_{4} \\
\leq & C \frac{e^{-\tau / 2}|a|}{\|U\|}\left(\int|y|^{2} U^{2} d y\right)^{1 / 2}+C e^{-\tau}|a|^{2} \\
& +e^{-\tau / 2}\left\|(A(\tau)-\bar{Q}(\tau) I) \frac{U}{\|U\|}\right\|\left(\frac{M_{1}}{\|U\|}(\|y U\|+\|\nabla U\|)+e^{-\tau / 2} M_{0}\right) .
\end{aligned}
$$

It is easy to check that

$$
\|\nabla U\|^{2}+\|y U\|^{2} \leq C\left(\bar{Q}(\tau)_{+}+e^{-\tau}|a|^{2}+1\right)\|U\|^{2}
$$

where

$$
x_{+}=\max \{x, 0\}, \quad x \in \mathbb{R} .
$$

Consequently,

$$
\begin{aligned}
\frac{1}{2} \bar{Q}^{\prime}(\tau)+ & \left\|(A(\tau)-\bar{Q}(\tau) I) \frac{U}{\|U\|}\right\|^{2} \\
\leq & C e^{-\tau / 2}|a|\left(\bar{Q}(\tau)_{+}+e^{-\tau}|a|^{2}+1\right)^{1 / 2}+C e^{-\tau}|a|^{2} \\
& +C e^{-\tau / 2}\left\|(A(\tau)-\bar{Q}(\tau) I) \frac{U}{\|U\|}\right\|\left(M_{1}\left(\bar{Q}(\tau)_{+}+e^{-\tau}|a|^{2}+1\right)^{1 / 2}+e^{-\tau / 2} M_{0}\right) .
\end{aligned}
$$

Applying Young's inequality to the last term, we get

$$
\begin{gathered}
\bar{Q}^{\prime}(\tau) \leq C e^{-\tau / 2}|a|\left(\bar{Q}(\tau)_{+}+e^{-\tau}|a|^{2}+1\right)^{1 / 2}+C e^{-\tau}|a|^{2} \\
+C e^{-\tau}\left(M_{1}^{2}\left(\bar{Q}(\tau)_{+}+e^{-\tau}|a|^{2}+1\right)+e^{-\tau} M_{0}^{2}\right) \\
\leq C e^{-\tau} M_{1}^{2} \bar{Q}(\tau)_{+}+C e^{-\tau / 2}|a|\left(\bar{Q}(\tau)_{+}\right)^{1 / 2}+C e^{-\tau / 2}|a| \\
+C e^{-\tau}\left(|a|^{2}+M_{1}^{2}\right)+C e^{-2 \tau}\left(M_{1}^{2}|a|^{2}+M_{0}^{2}\right) .
\end{gathered}
$$

Observe that

$$
C e^{-\tau_{0}} M_{1}^{2}=C e^{-\log (1 / \epsilon)} M_{1}^{2} \leq \frac{C M_{1}^{2}}{K_{0}\left(M_{1}^{2}+M_{0}^{2 / 3}\right)} \leq \frac{1}{2},
$$

where the last inequality is obtained by assuming that $K_{0}$ is a sufficiently large constant. Thus we may now apply [Ku1, Lemma 2.2] and estimate

$$
\bar{Q}(\tau) \leq C\left(\max \left(\bar{Q}\left(\tau_{0}\right), 0\right)+M_{1}^{2}|a|^{2} \epsilon^{2}+M_{1}^{2} \epsilon+M_{0}^{2} \epsilon^{2}+|a|^{2} \epsilon+|a| \epsilon^{1 / 2}\right), \quad \tau \geq \tau_{0} .
$$


By (3.12), (4.11), 4.12), and (4.13) we have

$$
\begin{aligned}
\bar{Q}\left(\tau_{0}\right) & \leq Q(\tau)+\left|\frac{e^{-\tau_{0} / 2} a_{j}}{\|U\|^{2}} \int y_{j} U^{2} d y\right| \\
& \leq 2 \epsilon q(\epsilon)+\frac{C \sqrt{\epsilon}}{\epsilon} \frac{\left(H U\left(\tau_{0}\right), U\left(\tau_{0}\right)\right)^{1 / 2}}{\left(U\left(\tau_{0}\right), U\left(\tau_{0}\right)\right)^{1 / 2}} \leq 2 \epsilon q(\epsilon)+\frac{C}{\sqrt{\epsilon}}(\epsilon q(-\epsilon))^{1 / 2} \\
& \leq 2 \epsilon q(\epsilon)+C q(-\epsilon)^{1 / 2} \leq 2 \epsilon q_{0}+C q_{0}^{1 / 2}
\end{aligned}
$$

where we used (2.5) in the last inequality. Recall that $a=-x_{\epsilon} / \epsilon$, where $x_{\epsilon} \in \Omega$, which implies $|a| \leq C / \epsilon$. Therefore,

$$
\begin{aligned}
\bar{Q}(\tau) & \leq C\left(\epsilon q_{0}+q_{0}^{1 / 2}+M_{1}^{2}+1+M_{0}^{2 / 3}+\sqrt{M_{1}^{2}+M_{0}^{2 / 3}}\right) \\
& \leq C\left(q_{0}+M_{1}^{2}+1+M_{0}^{2 / 3}\right) \leq C\left(M_{1}^{2}+1+M_{0}^{2 / 3}\right) \\
& =K_{M_{0}, M_{1}}
\end{aligned}
$$

by allowing $C$ to depend on $q_{0}$. Going back to (4.40) and applying Young's inequality one more time, we obtain

$$
\begin{aligned}
& \frac{1}{2} \bar{Q}^{\prime}(\tau)+\left\|(A(\tau)-\bar{Q}(\tau) I) \frac{U}{\|U\|}\right\| \|^{2} \\
& \leq C e^{-\tau / 2}|a|\left(\bar{Q}(\tau)_{+}+e^{-\tau}|a|^{2}+1\right)^{1 / 2}+C e^{-\tau}|a|^{2} \\
& \quad+C e^{-\tau} M_{1}^{2}\left(\bar{Q}(\tau)_{+}+e^{-\tau}|a|^{2}+1\right)+M_{0}^{2} e^{-2 \tau}
\end{aligned}
$$

Integrating (4.46) and using the bound on $\bar{Q}$, we get

$$
\begin{aligned}
& \frac{1}{2}\left(\bar{Q}(\tau)-\bar{Q}\left(\tau_{0}\right)\right)+\int_{\tau_{0}}^{\tau}\left\|(A(s)-\bar{Q}(s) I) \frac{U(\cdot, s)}{\|U(\cdot, s)\|}\right\|^{2} d s \\
& \leq C|a|\left(K_{M_{0}, M_{1}}^{1 / 2}\left(\epsilon^{1 / 2}-e^{-\tau / 2}\right)\right)+C|a|^{2}\left(\epsilon-e^{-\tau}\right) \\
& \quad+C\left(M_{1}^{2} K_{M_{0}, M_{1}}\left(\epsilon-e^{-\tau}\right)+M_{1}^{2}|a|^{2}\left(\epsilon^{2}-e^{-2 \tau}\right)\right)+C M_{0}^{2}\left(\epsilon^{2}-e^{-2 \tau}\right) .
\end{aligned}
$$

In order to estimate the second term on the left from below, let $\|v\|_{L^{2}}=1$. Then

$$
\|(A(s)-\bar{Q} I) v\|^{2} \geq \frac{1}{2}\|(H-\bar{Q} I) v\|^{2}-C e^{-\tau}|a|^{2} \bar{Q}-C e^{-\tau}|a|^{2} .
$$

Using this inequality in (4.46) and letting $\tau \rightarrow \infty$, we get

$$
\int_{\tau_{0}}^{\infty}\left\|(H-\bar{Q}(s) I) \frac{U(\cdot, s)}{\|U(\cdot, s)\|}\right\|^{2} d s<\infty .
$$

Combining this fact with (4.14) and

$$
\limsup _{\tau \rightarrow \infty} \bar{Q}^{\prime}(\tau) \leq 0
$$

which follows from (4.46), we get

$$
\operatorname{dist}(\bar{Q}(\tau), \operatorname{sp}(A(\tau))) \rightarrow 0
$$


as $\tau \rightarrow \infty$. Using (4.19), we finally obtain

$$
\bar{Q}(\tau) \rightarrow \frac{m}{2}
$$

as $\tau \rightarrow \infty$ for some $m \in \mathbb{N}_{0}$. Going back to [4.7), we have

$$
\left(\partial_{\tau} U, U\right)+\bar{Q}(\tau)=e^{-\tau / 2} a_{j}\left(\partial_{j} U, U\right)+e^{-\tau / 2}\left(y_{j} W_{j} U, U\right)+e^{-\tau / 2}\left(W_{j} \partial_{j} U, U\right)+e^{-\tau}(V U, U)
$$

which may be rewritten as

$$
\frac{1}{2} \frac{1}{\|U\|^{2}} \frac{d}{d \tau}\|U\|^{2}+\bar{Q}(\tau)=\frac{f(\tau)}{\|U\|^{2}}
$$

where

$$
\frac{f(\tau)}{\|U\|^{2}}=e^{-\tau / 2} a_{j} \frac{\left(\partial_{j} U, U\right)}{\|U\|^{2}}+e^{-\tau / 2} \frac{\left(y_{j} W_{j} U, U\right)}{\|U\|^{2}}+e^{-\tau / 2} \frac{\left(W_{j} \partial_{j} U, U\right)}{\|U\|^{2}}+e^{-\tau} \frac{(V U, U)}{\|U\|^{2}} .
$$

Note that $\left(\partial_{j} U, U\right)=0$, and thus

$$
\begin{aligned}
\frac{f(\tau)}{\|U\|^{2}} & \leq e^{-\tau / 2} M_{1}\left(\frac{\int|y| U^{2} d y+\int|\nabla U||U| d s}{\|U\|^{2}}\right)+e^{-\tau} M_{0} \\
& \leq 2 e^{-\tau / 2} M_{1}\left(\frac{\int|y| U^{2} d y+\int|\nabla U|^{2} d s+\int|U|^{2} d s}{\|U\|^{2}}\right)+e^{-\tau} M_{0} \\
& \leq C e^{-\tau / 2} M_{1}\left(|\bar{Q}(\tau)|+e^{-\tau}|a|^{2}+1\right)+e^{-\tau} M_{0} .
\end{aligned}
$$

As $\bar{Q}(\tau)$ is uniformly bounded,

$$
\int_{\tau_{1}}^{\tau} \frac{f(s) d s}{\|U(\cdot, s)\|^{2}} \leq C K_{M_{0}, M_{1}} M_{1}\left(e^{-\tau_{1} / 2}-e^{-\tau / 2}\right)+C M_{1}|a|^{2}\left(e^{-3 \tau_{1} / 2}-e^{-3 \tau / 2}\right)+M_{0}\left(e^{-\tau_{1}}-e^{-\tau}\right) .
$$

Integrating (4.53), we get

$$
\begin{aligned}
\frac{1}{2} \log & \|U(\cdot, \tau)\|^{2}-\frac{1}{2} \log \left\|U\left(\cdot, \tau_{1}\right)\right\|^{2} \\
= & -\int_{\tau_{1}}^{\tau} \bar{Q}(s) d s+\int_{\tau_{1}}^{\tau} \frac{f(s)}{\|U(\cdot, s)\|^{2}} d s \\
& =-\frac{m}{2}\left(\tau-\tau_{1}\right)-\int_{\tau_{1}}^{\tau}\left(\bar{Q}(s)-\frac{m}{2}\right) d s+\mathcal{O}\left(e^{-\tau_{1} / 2}-e^{-\tau / 2}\right), \text { as } \tau \rightarrow \infty .
\end{aligned}
$$

By (4.47), we have that for all $\delta>0$ there exists $t_{1}(\delta)>0$ such that

$$
-\delta\left(\tau-\tau_{1}\right) \leq \log \|U(\tau)\|^{2}-\log \left\|U\left(\tau_{1}\right)\right\|^{2}+m\left(\tau-\tau_{1}\right) \leq \delta\left(\tau-\tau_{1}\right), \quad \tau \geq \tau_{1}(\delta) .
$$

Therefore,

$$
e^{-\delta\left(\tau-\tau_{1}\right)} \leq \frac{e^{\tau m}\|U(\tau)\|^{2}}{e^{\tau_{1} m}\left\|U\left(\tau_{1}\right)\right\|^{2}} \leq e^{\delta\left(\tau-\tau_{1}\right)}, \quad \tau \geq \tau_{1} .
$$

Consequently, for all $\delta>0$, there exists $\tau_{1} \geq \tau_{0}$ such that

$$
A_{1}(\delta) e^{-\delta \tau} \leq e^{m \tau}\|U(\tau)\|^{2} \leq A_{2}(\delta) e^{\delta \tau}, \quad \tau \geq \tau_{1}
$$


for some positive constants $A_{1}(\delta)$ and $A_{2}(\delta)$. Switching back to the original variables, we obtain (4.24), and the proof is concluded.

A function $f(x, t)$ is homogeneous of degree $d$ if for any $\lambda>0$ and $(x, t) \in \mathbb{R}^{n} \times \mathbb{R} \backslash\{(0,0)\}$ if it satisfies

$$
f\left(\lambda x, \lambda^{2} t\right)=\lambda^{d} f(x, t) .
$$

A polynomial $P(x, t)$ of degree at most $d \in \mathbb{N}$ can be decomposed into a sum of homogeneous polynomials whose degree of homogeneity is at most $d$. The following elementary results regarding polynomials play an important role in the subsequent argument.

Lemma 4.2. Let $Q=\sum_{|\mu|+2 l=d} C_{\mu, l} x^{\mu} t^{l}$ be a homogeneous polynomial of degree $d \in \mathbb{N}_{0}$. Then

$$
\int_{\mathbb{R}^{n}} Q(x, t) G_{0}(x, t) d x=C_{0}|t|^{d / 2}
$$

where $C_{0}$ is a constant depending on the polynomial.

Proof of Lemma 4.2. By substituting $Q$ in the integral, we obtain

$$
\begin{array}{rl}
\int_{\mathbb{R}^{n}} & Q(x, t) G_{0}(x, t) d x=\sum_{|\mu|+2 l=d} C_{\mu, l} \int_{\mathbb{R}^{n}} x^{\mu} t^{l} \frac{e^{-|x|^{2} / 4|t|}}{|t|^{n / 2}} d x \\
& =\sum_{|\mu|+2 l=d} C_{\mu, l}|t|^{l+|\mu| / 2} \int_{\mathbb{R}^{n}} \frac{x^{\mu}}{|t|^{|\mu| / 2}} \frac{e^{-|x|^{2} / 4|t|}}{|t|^{n / 2}} d x \\
& =\sum_{|\mu|+2 l=d} C_{\mu, l}|t|^{l+|\mu| / 2} \int_{\mathbb{R}} \cdots \int_{\mathbb{R}}\left(\frac{x_{1}}{|t|^{1 / 2}}\right)^{\mu_{1}} \cdots\left(\frac{x_{n}}{|t|^{1 / 2}}\right)^{\mu_{n}} \frac{e^{-|x|^{2} / 4|t|}}{|t|^{n / 2}} d x_{1} \cdots d x_{n} \\
& =|t|^{d / 2} \sum_{|\mu|+2 l=d} C_{\mu, l} \Gamma\left(\mu_{1}+1\right) \cdots \Gamma\left(\mu_{n}+1\right)
\end{array}
$$

and the lemma is established.

Lemma 4.3. Consider the polynomial $x^{\mu} t^{l}$ with $|\mu|+2 l=2 d$ for some integer $d \geq 0$. If all the coordinates of $\mu=\left(\mu_{1}, \mu_{2}, \ldots, \mu_{n}\right)$ are even, then for all $r>0$

$$
\int_{B(0, r)} x^{\mu} t^{l} G_{0}(x, t) d x=C_{0}|t|^{d}+\mathcal{O}\left(|t|^{d+1}\right), \text { as } t \rightarrow 0-
$$

where $C_{0}$ is a constant. If $\mu_{i}$ is an odd integer for some $i \in\{1, \cdots, n\}$, then

$$
\int_{B(0, r)} x^{\mu} t^{l} G_{0}(x, t) d x=0 .
$$


Proof of Lemma 4.3. For $|\mu|>0$, we have

$$
\begin{aligned}
& \left|\int_{B(0, r)} x^{\mu} t^{l} G_{0}(x, t) d x\right| \\
& \quad \leq C|t|^{l+|\mu| / 2} \prod_{i=1}^{n} \int_{0}^{r}\left(\frac{x_{i}}{|t|^{1 / 2}}\right)^{\mu_{i}} \frac{e^{-\left|x_{i}\right|^{2} / 4|t|}}{|t|^{1 / 2}} d x_{i} \\
& \quad=K|t|^{d} \prod_{i=1}^{n} \int_{0}^{r^{2} / 4|t|} z^{\mu_{i} / 2-1 / 2} e^{-z} d z
\end{aligned}
$$

where $K$ is a fixed constant. It is easy to check that

$$
\left|C_{0}-K \prod_{i=1}^{n} \int_{0}^{r^{2} / 4|t|} z^{\mu_{i} / 2-1 / 2} e^{-z} d z\right|=\mathcal{O}(|t|), \quad \text { as } t \rightarrow 0-
$$

where

$$
C_{0}=K \prod_{i=1}^{n} \int_{0}^{\infty} z^{\mu_{i} / 2-1 / 2} e^{-z} d z
$$

and (4.56) follows. If $\mu_{i}$ is odd for some $i$, then we have (4.57) by symmetry.

\section{The proof of the main theorem}

Before concluding with the proof of the main theorem, we need a statement connecting the order of vanishing with the degree of the eigenfunction.

Lemma 5.1. Consider a solution $u \in W_{\infty}^{2,1}(\Omega \times I)$ to the equation

$$
\partial_{t} u+\Delta u=a_{j} \partial_{j} u+w_{j}(x, t) \partial_{j} u+v(x, t) u
$$

for $t \in[-\epsilon, 0]$ with $\epsilon \in(0,1)$ where $a_{j}$ are constants and where $w_{j}$ and $v$ satisfy (2.2) and (2.3), and suppose that $u$ has finite order of vanishing $d \in \mathbb{N}_{0}$ at $(0,0)$. With $m \in \mathbb{N}$, assume that for all $\delta>0$ there exist $\eta \in(0, \epsilon]$ and $A_{1}(\delta), A_{2}(\delta)>0$ such that

$$
A_{1}(\delta)|t|^{m+\delta} \leq \int_{\mathbb{R}^{n}} u^{2}(x, t) G_{0}(x, t) d x \leq A_{2}(\delta)|t|^{m-\delta}, \quad t \in[-\eta, 0] .
$$

Then $d=m$.

Proof of Lemma 5.1. Since the statement clearly holds when $d=0$, we assume $d \in \mathbb{N}$. First, assume that (5.2) holds for some fixed $\delta>0$. Fix $\delta>0$. Since the degree of vanishing of $u$ at $(0,0)$ is $d>0$, there exists $\gamma>0$ such that

$$
|u(x, t)| \leq \gamma|(x, t)|^{d}=\gamma\left(|x|^{2}+|t|\right)^{d / 2}
$$

for all $(x, t) \in Q_{1}$. Note that for any $R>0$ we have

$$
\int_{\mathbb{R}^{n} \backslash B(0, R)} u^{2}(x, t) G_{0}(x, t) d x \leq\|u\|_{L^{\infty}\left(\mathbb{R}^{n}\right)}^{2} \int_{R}^{\infty} \int_{\partial B(0, r)} \frac{e^{-r^{2} / 4|t|}}{|t|^{n / 2}} d S_{y} d r \leq C\|u\|_{L^{\infty}}^{2} \frac{|t|^{1 / 2}}{R} e^{-R^{2} / 8|t|} .
$$


By (5.2) and (5.4), for all $\delta>0$ there exist $\eta \in(0, \epsilon)$ and $A_{1}(\delta), A_{2}(\delta)>0$ such that

$$
\frac{1}{2} A_{1}(\delta)|t|^{m+\delta} \leq \int_{B(0, R)} u^{2}(x, t) G_{0}(x, t) d x \leq A_{2}(\delta)|t|^{m-\delta}, \quad t \in[-\eta, 0] .
$$

Furthermore, by Lemmas 4.2 and 4.3

$$
\int_{B(0, R)} u^{2}(x, t) G_{0}(x, t) d x \leq C \gamma^{2} \int_{B(0, R)}\left(|x|^{2}+|t|\right)^{d} G_{0}(x, t) d x \leq C|t|^{d}
$$

where $C$ depends on $R$. Combining this with (5.5), we get

$$
\frac{1}{2} A_{1}(\delta)|t|^{m+\delta} \leq C|t|^{d}, \quad t \in[-\eta, 0]
$$

which yields

$$
d \leq m+\delta
$$

For the other direction, we use [AV, Theorem 1.1] on a structure of a solution in a neighborhood of a point where $u$ vanishes. Recall that $u$ solves

$$
\partial_{t} u-\Delta u=a_{j} \partial_{j} u+w_{j}(x, t) \partial_{j} u+v(x, t) u=f(x, t),
$$

and for all $(x, t) \in Q_{1}$,

$$
\begin{aligned}
|f(x, t)| & \leq\left(|a|+\|w\|_{L^{\infty}(\Omega \times I)}\right) \max _{j}\left|\partial_{j} u(x, t)\right|+\|v(x, t)\|_{L^{\infty}(\Omega \times I)}|u(x, t)| \\
& \leq C|(x, t)|^{d-1}
\end{aligned}
$$

where $C$ depends on $u, v$, and $w$. This implies that for any $r \leq 1$, and $\alpha \in(0,1)$

$$
\|f\|_{L^{\infty}\left(Q_{r}\right)} \leq C r^{d-1}
$$

Therefore, by [AV, Theorem 1.1], for the solution $u \in W_{\infty}^{2,1}\left(Q_{1}\right)$ of $\left(\partial_{t}+\Delta\right) u=f$, there exists a homogeneous caloric polynomial $P_{d}$ of degree less than or equal to $d$ such that for any $\alpha \in(0,1)$,

$$
\left|u(x, t)-P_{d}(x, t)\right| \leq C|(x, t)|^{d+\alpha}
$$

for $(x, t) \in Q_{1}$. Since the degree of vanishing of $u$ is $d$, the degree of $P_{d}$ has to be $d$. Thus we may write

$$
P_{d}=\sum_{|\mu|+2 l=d} C_{\mu, l} x^{\mu} t^{l}
$$

where not all $C_{\mu, d}$ equal zero. Then, by (5.2) and (5.9), we have for all $t \in[-\eta, 0]$,

$$
\begin{aligned}
\int_{B(0,1)} & P_{d}^{2} G_{0}(x, t) d x \\
\leq & C \int_{B(0,1)} u^{2} G_{0}(x, t) d x+C \int_{B(0,1)}\left(|x|^{2}+|t|\right)^{d+\alpha} G_{0}(x, t) d x \\
\leq & C|t|^{m-\delta}+C \int_{\mathbb{R}^{n}}\left(|x|^{2}+|t|\right)^{d+\alpha} G_{0}(x, t) d x \\
= & C_{1}|t|^{m-\delta}+\mathcal{O}\left(|t|^{d+\alpha}\right) .
\end{aligned}
$$


On the other hand,

$$
\int_{B(0,1)} P_{d}^{2} G_{0}(x, t) d x=\sum_{|\mu|+2 l=2 d} C_{\mu, l} \int_{B(0,1)} x^{\mu}|t|^{l} G_{0}(x, t) d x=C_{0}|t|^{d}+\mathcal{O}\left(|t|^{d+1}\right), \text { as } t \rightarrow 0-
$$

by Lemma 4.3 where $C_{0}>0$. Consequently, we have

$$
C_{0}|t|^{d}+\mathcal{O}\left(|t|^{d+1}\right) \leq C_{1}|t|^{m-\delta}+C|t|^{d+\alpha}, \text { as } t \rightarrow 0-
$$

which implies

$$
d \geq m-\delta
$$

By (5.7) and (5.12), we get

$$
m-\delta \leq d \leq m+\delta
$$

and since this holds for all $\delta>0$, we conclude that $d=m$.

Proof of Theorem [2.1. In Lemmas 4.1 and 5.1 we have shown that

$$
\bar{Q}(\tau) \leq C\left(M_{1}^{2}+M_{0}^{2 / 3}\right)
$$

for all $\tau \in\left[\tau_{0}, \infty\right)$, and

$$
\bar{Q}(\tau) \rightarrow \frac{m}{2},
$$

as $\tau \rightarrow \infty$, where $m=O_{(0,0)}(u)$. Combining these two facts, we arrive at the desired conclusion.

\section{The case $\mathbb{R}^{n}$}

Here we present the modifications when the periodicity assumption is removed. Let $u$ be a solution of (2.1) defined for $(x, t) \in \mathbb{R}^{n} \times I$, where $I$ is an open interval containing $\left[T_{0}, T_{0}+T\right]$. Instead of periodicity, we assume (2.7), where $C$ is a constant. The coefficients $v$ and $w$ satisfy (2.2) and (2.3). For simplicity, we assume $M_{0}, M_{1} \geq 1$. Let $q_{0}$ be an upper bound for the Dirichlet quotients of $u$ on $\left[T_{0}, T_{0}+T\right]$, i.e.,

$$
\frac{\|\nabla u(\cdot, t)\|_{L^{2}}^{2}}{\|u(\cdot, t)\|_{L^{2}}^{2}} \leq q_{0}, \quad t \in\left[T_{0}, T_{0}+T\right]
$$

The following is the analog of Theorem 2.1

Theorem 6.1. Let $u \in W_{\infty}^{2,1}(\Omega \times I)$ be a nontrivial solution of (2.1) with $w_{j}$ and $v$ satisfying (2.2) and (2.3), respectively. Then the order of vanishing of $u$ at $\left(0, T_{0}+T\right)$ satisfies

$$
O_{\left(0, T_{0}+T\right)}(u) \leq C\left(M_{1}^{2}+M_{0}^{2 / 3}\right)
$$

where the constant depends on $T, M$, and $q_{0}$. 
The proof is the same as in the periodic case except that we need to modify Lemma 3.1 stated next. Assume, as above, that $T_{0}=-T$ so that the time interval is $[-T, 0]$.

Lemma 6.2. Let $u$ be as above with $v$ and $w_{j}$ satisfying (2.2) and (2.3). Let $\epsilon \in(0, T)$ be such that

$$
\epsilon \leq \frac{1}{C}
$$

for a sufficiently large constant $C$. Then

$$
\frac{\epsilon \int_{\mathbb{R}^{n}}\left|\nabla u\left(x_{\epsilon}+y,-\epsilon\right)\right|^{2} G_{0}(y,-\epsilon) d y}{\int_{\mathbb{R}^{n}} u\left(x_{\epsilon}+y,-\epsilon\right)^{2} G_{0}(y,-\epsilon) d y} \leq C M \epsilon q(-\epsilon)
$$

for some $x_{\epsilon} \in B_{2}$.

Proof of Lemma 6.2. Assume that, for some $\lambda>0$, we have

$$
\lambda \int_{\mathbb{R}^{n}} u(x+y,-\epsilon)^{2} G_{0}(y,-\epsilon) d y \leq \int_{\mathbb{R}^{n}}|\nabla u(x+y,-\epsilon)|^{2} G_{0}(y,-\epsilon) d y, \quad x \in B_{2} .
$$

We shall show that this cannot hold if $\lambda \geq C M q(-\epsilon)$ for a sufficiently large constant $C>0$. We integrate (6.5) in $x$ over $B_{2}$ and change the order of integration to obtain

$$
\int_{\mathbb{R}^{n}} G_{0}(y,-\epsilon) d y \int_{B_{2}} u(x+y,-\epsilon)^{2} d x \leq \frac{1}{\lambda} \int_{\mathbb{R}^{n}} G_{0}(y,-\epsilon) d y \int_{B_{2}}|\nabla u(x+y,-\epsilon)|^{2} d x
$$

Note that the right side of (6.6) is bounded from above by

$$
\frac{1}{\lambda}\|\nabla u(\cdot,-\epsilon)\|_{L^{2}}^{2} \int G_{0}(y,-\epsilon) d y=\frac{(2 \pi)^{n / 2}}{\lambda}\|\nabla u(\cdot,-\epsilon)\|_{L^{2}}^{2}
$$

while the left side of (6.6) equals

$$
\begin{aligned}
\int_{\mathbb{R}^{n}} & G_{0}(y,-\epsilon) d y \int_{B_{2}(y)} u(x,-\epsilon)^{2} d x \\
& =\int_{B_{1 / 2}} G_{0}(y,-\epsilon) d y \int_{B_{2}(y)} u(x,-\epsilon)^{2} d x+\int_{\mathbb{R}^{n} \backslash B_{1 / 2}} G_{0}(y,-\epsilon) d y \int_{B_{2}(y)} u(x,-\epsilon)^{2} d x \\
& \geq \int_{B_{1 / 2}} G_{0}(y,-\epsilon) d y \int_{B_{2}(y)} u(x,-\epsilon)^{2} d x .
\end{aligned}
$$

The double integral on the far right side is bounded from below by

$$
\begin{aligned}
\int_{B_{1 / 2}} & G_{0}(y,-\epsilon) d y \int_{B_{1}(0)} u(x,-\epsilon)^{2} d x \\
& \geq \frac{1}{2}(2 \pi)^{n / 2} \int_{B_{1}(0)} u(x,-\epsilon)^{2} d x \geq \frac{1}{C M} \int_{\mathbb{R}^{n}} u(x,-\epsilon)^{2} d x
\end{aligned}
$$

where the first inequality holds by (6.3) if $C$ is sufficiently large and the second one holds by (2.7). Therefore, we get

$$
\|u(x,-\epsilon)\|_{L^{2}}^{2} \leq \frac{C M}{\lambda}\|\nabla u(\cdot,-\epsilon)\|_{L^{2}} .
$$

Using (6.1), we get a contradiction if $\lambda \geq C M q(-\epsilon)$. 


\section{Acknowledgments}

The authors were supported in part by the NSF grants DMS-1311943 and DMS-1615239.

\section{References}

[A] S. Agmon, Unicité et convexité dans les problèmes différentiels, Séminaire de Mathématiques Supérieures, No. 13 (Été, 1965), Les Presses de l’Université de Montréal, Montreal, Que., 1966.

[AN] S. Agmon and L. Nirenberg, Lower bounds and uniqueness theorems for solutions of differential equations in a Hilbert space, Comm. Pure Appl. Math. 20 (1967), 207-229.

[AE] G. Alessandrini and L. Escauriaza, Null-controllability of one-dimensional parabolic equations, ESAIM Control Optim. Calc. Var. 14 (2008), no. 2, 284-293.

[AMRV] G. Alessandrini, A. Morassi, E. Rosset, and S. Vessella, On doubling inequalities for elliptic systems, J. Math. Anal. Appl. 357 (2009), no. 2, 349-355.

[Al] F.J. Almgren, Jr., Dirichlet's problem for multiple valued functions and the regularity of mass minimizing integral currents, Minimal submanifolds and geodesics (Proc. Japan-United States Sem., Tokyo, 1977), North-Holland, Amsterdam-New York, 1979, pp. 1-6.

[An] S. Angenent, The zero set of a solution of a parabolic equation, J. Reine Angew. Math. 390 (1988), 79-96.

[AV] G. Alessandrini and S. Vessella, Local behaviour of solutions to parabolic equations, Comm. Partial Differential Equations 13 (1988), no. 9, 1041-1058.

[BC] L. Bakri and J.-B. Casteras, Quantitative uniqueness for Schrödinger operator with regular potentials, Math. Methods Appl. Sci. 37 (2014), no. 13, 1992-2008.

[B1] L. Bakri, Carleman estimates for the Schrödinger operator. Applications to quantitative uniqueness, Comm. Partial Differential Equations 38 (2013), no. 1, 69-91.

[B2] L. Bakri, Quantitative uniqueness for Schrödinger operator, Indiana Univ. Math. J. 61 (2012), no. 4, 1565-1580.

[BK] J. Bourgain and C.E. Kenig, On localization in the continuous Anderson-Bernoulli model in higher dimension, Invent. Math. 161 (2005), no. 2, 389-426.

[CRV] B. Canuto, E. Rosset, and S. Vessella, Quantitative estimates of unique continuation for parabolic equations and inverse initial-boundary value problems with unknown boundaries, Trans. Amer. Math. Soc. 354 (2002), no. 2, 491-535.

[C] T. Carleman, Sur un problème d'unicité pur les systèmes d'équations aux dérivées partielles à deux variables indépendantes, Ark. Mat., Astr. Fys. 26 (1939), no. 17, 9. 
[Ch] X.-Y. Chen, A strong unique continuation theorem for parabolic equations, Math. Ann. 311 (1998), no. 4, 603-630.

[CFNT] P. Constantin, C. Foias, B. Nicolaenko, and R. Temam, Spectral barriers and inertial manifolds for dissipative partial differential equations, J. Dynam. Differential Equations 1 (1989), no. 1, $45-73$.

[DF1] H. Donnelly and C. Fefferman, Nodal sets of eigenfunctions on Riemannian manifolds, Invent. Math. 93 (1988), no. 1, 161-183.

[DF2] H. Donnelly and C. Fefferman, Growth and geometry of eigenfunctions of the Laplacian, Analysis and partial differential equations, Lecture Notes in Pure and Appl. Math., vol. 122, Dekker, New York, 1990, pp. 635-655.

[DF3] H. Donnelly and C. Fefferman, Nodal sets for eigenfunctions of the Laplacian on surfaces, J. Amer. Math. Soc. 3 (1990), no. 2, 333-353.

[EF] L. Escauriaza and F.J. Fernández, Unique continuation for parabolic operators, Ark. Mat. 41 (2003), no. 1, 35-60.

[EFV] L. Escauriaza, F. J. Fernández, and S. Vessella, Doubling properties of caloric functions, Appl. Anal. 85 (2006), no. 1-3, 205-223.

[EV] L. Escauriaza and L. Vega, Carleman inequalities and the heat operator. II, Indiana Univ. Math. J. 50 (2001), no. 3, 1149-1169.

[EVe] L. Escauriaza and S. Vessella, Optimal three cylinder inequalities for solutions to parabolic equations with Lipschitz leading coefficients, Inverse problems: theory and applications (Cortona/Pisa, 2002), Contemp. Math., vol. 333, Amer. Math. Soc., Providence, RI, 2003, pp. $79-$ 87.

[FS] C. Foias and J.-C. Saut, Asymptotic behavior, as $t \rightarrow \infty$, of solutions of the Navier-Stokes equations, Nonlinear partial differential equations and their applications. Collège de France Seminar, Vol. IV (Paris, 1981/1982), Res. Notes in Math., vol. 84, Pitman, Boston, MA, 1983, pp. $74-86$.

[GL] N. Garofalo and F.-H. Lin, Monotonicity properties of variational integrals, $A_{p}$ weights and unique continuation, Indiana Univ. Math. J. 35 (1986), no. 2, 245-268.

[H1] Q. Han, Schauder estimates for elliptic operators with applications to nodal sets, J. Geom. Anal. 10 (2000), no. 3, 455-480.

[H2] Q. Han, On the Schauder estimates of solutions to parabolic equations, Ann. Scuola Norm. Sup. Pisa Cl. Sci. (4) 27 (1998), no. 1, 1-26 (1999).

[JK] D. Jerison and C.E. Kenig, Unique continuation and absence of positive eigenvalues for Schrödinger operators, Ann. of Math. (2) 121 (1985), no. 3, 463-494, With an appendix by E. M. Stein. 
[K1] C.E. Kenig, Restriction theorems, Carleman estimates, uniform Sobolev inequalities and unique continuation, Harmonic analysis and partial differential equations (El Escorial, 1987), Lecture Notes in Math., vol. 1384, Springer, Berlin, 1989, pp. 69-90.

[K2] C.E. Kenig, Some recent applications of unique continuation, Recent developments in nonlinear partial differential equations, Contemp. Math., vol. 439, Amer. Math. Soc., Providence, RI, 2007, pp. 25-56.

[K3] C.E. Kenig, Quantitative unique continuation, logarithmic convexity of Gaussian means and Hardy's uncertainty principle, Perspectives in partial differential equations, harmonic analysis and applications, Proc. Sympos. Pure Math., vol. 79, Amer. Math. Soc., Providence, RI, 2008, pp. 207-227.

[KSW] C. Kenig, L. Silvestre, and J.-N. Wang, On Landis' conjecture in the plane, Comm. Partial Differential Equations 40 (2015), no. 4, 766-789.

[Ku1] I. Kukavica, Quantitative uniqueness for second-order elliptic operators, Duke Math. J. 91 (1998), no. 2, 225-240.

[Ku2] I. Kukavica, Quantitative uniqueness and vortex degree estimates for solutions of the GinzburgLandau equation, Electron. J. Differential Equations (2000), No. 61, 15 pp. (electronic).

[Ku3] I. Kukavica, Self-similar variables and the complex Ginzburg-Landau equation, Comm. Partial Differential Equations 24 (1999), no. 3-4, 545-562.

[Ku4] I. Kukavica, Length of vorticity nodal sets for solutions of the $2 D$ Navier-Stokes equations, Comm. Partial Differential Equations 28 (2003), no. 3-4, 771-793.

[Kur] K. Kurata, On a backward estimate for solutions of parabolic differential equations and its application to unique continuation, Spectral and scattering theory and applications, Adv. Stud. Pure Math., vol. 23, Math. Soc. Japan, Tokyo, 1994, pp. 247-257.

[KT] H. Koch and D. Tataru, Carleman estimates and unique continuation for second order parabolic equations with nonsmooth coefficients, Comm. Partial Differential Equations 34 (2009), no. 4-6, 305-366.

[L] F.-H. Lin, Nodal sets of solutions of elliptic and parabolic equations, Comm. Pure Appl. Math. 44 (1991), no. 3, 287-308.

[LNW] C.-L. Lin, G. Nakamura, and Jenn-Nan Wang, Quantitative uniqueness for second order elliptic operators with strongly singular coefficients, Rev. Mat. Iberoam. 27 (2011), no. 2, 475-491.

[M] V.Z. Meshkov, On the possible rate of decrease at infinity of the solutions of second-order partial differential equations, Mat. Sb. 182 (1991), no. 3, 364-383.

[P] C.-C. Poon, Unique continuation for parabolic equations, Comm. Partial Differential Equations 21 (1996), no. 3-4, 521-539. 
[V] S. Vessella, Carleman estimates, optimal three cylinder inequalities and unique continuation properties for parabolic operators, Progress in analysis, Vol. I, II (Berlin, 2001), World Sci. Publ., River Edge, NJ, 2003, pp. 485-492. 